\title{
THE GENERALIZED BELTRAMI PROBLEM CONCERNING
}

\section{GEODESIC REPRESENTATION*}

\author{
BY \\ EDWARD KASNER
}

In 1865 BeLtrami showed that the only surfaces which can be represented point by point upon the plane in such a manner that the geodesics of the surface are represented by straight lines are the surfaces of constant curvature. $\dagger$ A natural extension is to consider instead of the straight lines any doubly infinite system of curves

$$
F^{\prime}(x, y, \lambda, \mu)=0,
$$

and to investigate the existence and the properties of the surfaces which can be built upon the plane in such a manner that the geodesics are represented by the curves of the prescribed system. This extension was proposed by Dr. STECKER in two papers published in preceding volumes of these Transactions; $\ddagger$ but the only case actually considered by him is that of a linear system

$$
\lambda \phi(x, y)+\mu \psi(x, y)+\chi(x, y)=0 \text {. }
$$

Later Dr. Pell, $\S$ in a paper read at the summer meeting of the American Mathematical Society, 1902, investigated the same case of a linear system, employing Darboux's method based upon the inverse problem of the calculus of variations. Neither Dr. Stecker nor Dr. Pell arrive at a solution of the problem proposed.

As a matter of fact the extension of Beltrami's problem to a linear system (2) is trivial. For by the point of transformation

the system (2) becomes

$$
x_{1}=\frac{\phi(x, y)}{\chi(x, y)}, \quad y_{1}=\frac{\psi(x, y)}{\chi(x, y)},
$$

$$
\lambda x_{1}+\mu y_{1}+1=0 \text {. }
$$

* Presented to the Society December 30, 1902. Received for publication November 26, 1902. † Beltrami, Annali di Matematica, vol. 7 (1866), pp. 185-201; Bianchi, Differentialgeometrie, German translation of LUK AT, § 242.

$\ddagger$ Vol. 2 , pp. $152-165$; vol. 3 , pp. 12-22.

8 Cf. abstract in the Bulletin of the A merican Mathematical Society, vol. 9 (1902), p. 93. 
That is, any surface which can be built upon the plane in such a manner that its geodesics are represented by (2), can also be built upon the plane so that the geodesics are.represented by the straight lines (3). Therefore the surface must be one of constant curvature. Conversely, any surface of constant curvature can be built upon the plane so that its geodesics are represented by any prescribed linear system (2); in fact, the representation is possible in $\infty^{8}$ distinct ways, since this is true of the equivalent system (3).*

Of course the representations considered are not in general conformal. If this condition be imposed, as is done in Dr. STECKER's papers, the problem is more specialized but may still be solved by simple considerations. The surface must be of constant curvature, as was seen above, but the form of the linear system of curves is no longer arbitrary; its particular form depends upon whether the curvature is zero or positive or negative. Any surface of constant curvature $f$ can be built conformally upon the plane so that the geodesics are represented by a linear system of circles, which for the three cases considered may be written respectively as follows:

$$
\begin{aligned}
& \lambda x_{1}+\mu y_{1}+x_{1}^{2}+y_{1}^{2}=0, \\
& \lambda x_{1}+\mu y_{1}+x_{1}^{2}+y_{1}^{2}-1=0, \\
& \lambda x_{1}+\mu y_{1}+x_{1}^{2}+y_{1}^{2}+1=0 .
\end{aligned}
$$

In the first case the circles all go through the origin, in the second they are orthogonal to the imaginary circle $x_{1}^{2}+y_{1}^{2}+1=0$, in the third they are orthogonal to the real circle $x_{1}^{2}+y_{1}^{2}-1=0$.

The most general conformal representation is obtained from the special representation just given by means of the general conformal transformation of the plane, which may be written

$$
x_{1}=\Phi(x, y), \quad y_{1}=\Psi(x, y),
$$

where $\Phi$ and $\Psi$ are any two conjugate harmonic functions, or, what is equivalent, where $\Phi+i \Psi$ is a function of the complex variable $x+i y$. The systems of

* Cf. Darboux, Théorie des Surfaces, vol. 3, p. 59.

† Branchi, Differentialgeometrie, Lukat's translation, $\$ 230$, where the result is stated for pseudospherical surfaces. Any surface of constant positive curvature may be appled to a sphere, whose stereographic projection gives the conformal transformation by which the geodesics become the circles $\left(4_{+}\right)$. The system $\left(4_{0}\right)$ for developable surfaces is obtained from the straight lines, representing the geodesics when the surface is developed on the plane, by inversion with respect to the unit circle. Cf. BUSSE, Inaugural-dissertation, Berlin, 1896, where it is proved that the only surfaces capable of conformal representation upon a plane so that the geodesics are represented by circles are the surfaces of constant currature. 
eircles representing the geodesics become by this transformation linear systems of curves :

$$
\begin{aligned}
& \lambda \Phi+\mu \Psi+\Phi^{2}+\Psi^{2}=0, \\
& \lambda \Phi+\mu \Psi+\Phi^{2}+\Psi^{2}-1=0, \\
& \lambda \Phi+\mu \Psi+\Phi^{2}+\Psi^{2}+1=0 .
\end{aligned}
$$

To sum up: The only surfaces capable of conformal representation upon a plane in such a manner that the geodesics are represented by a linear system of curves are the surfaces of constant curvature; according as the curvature is zero or positive or negative the linear system is of form $\left(6_{0}\right)$ or $\left(6_{+}\right)$or (6_). Conversely, if a system of form $\left(6_{0}\right)$ [or $\left(6_{+}\right)$or $\left.\left(6_{-1}\right)\right]$ is given, any surface of constant zero [or positive or negative] curvature can be built conformally upon the plane so that its geodesics are represented by the assigned system. *

Let us consider now the real extension of BeLtrami's problem proposed at the beginning of the paper: Given any two-parametersy stem of curves, represented either in the integral form

$$
F(x, y, \lambda, \mu)=0,
$$

or by the corresponding differential equation

$$
y^{\prime \prime}=f\left(x, y, y^{\prime}\right),
$$

we ask in the first place whether surfaces exist which can be built upon the plane so that the geodesics are represented by the assigned curves, and in the second place, if the surfaces exist, how may they be determined.

The differential equation of the geodesics on a surface whose first fundamental form is

may be written $\dagger$

$$
d s^{2}=E d x^{2}+2 F d x d y+G d y^{2}
$$

$$
\begin{aligned}
2\left(E G-F^{2}\right) y^{\prime \prime} & =\left(2 G F_{y}-G G_{x}-F G_{y}\right) y^{\prime 3} \\
& +\left(2 G E_{y}+2 F F_{y}-3 F G_{x}-E G_{y}\right) y^{\prime 2} \\
& +\left(G E_{x}+3 F E_{y}-2 F F_{x}-2 E G_{x}\right) y^{\prime} \\
& +\left(F E_{x}+E E_{y}-2 E F_{x}\right) .
\end{aligned}
$$

* The conformal representation is possible moreover in $\infty^{4}$ or $\infty^{3}$ ways according as the surface is developable or not. This is seen by considering the sub-groups of the six parameter group of circular (MöвIUS) transformations for which the linear systems (4) are invariant.

† Branchi, $l$. c., p. 154. The Gaussian coördinates on the surface are $x, y$ instead of the more usual $u, v$. 
Comparing (7) and (9), it is seen that in general, i. e., for an arbitrary system, no solution of the problem exists; for it is necessary in the first place that the differential equation (7) should be of the form

$$
y^{\prime \prime}=A y^{\prime 3}+B y^{\prime 2}+C y^{\prime}+D,
$$

where $A, B, C, D$ denote functions of $x$ and $y$. These functions, moreover, are not arbitrary, since they are expressible in terms of three functions $E, F, G$ :

$$
\begin{aligned}
& A=\frac{2 G F_{y}-G G_{x}-F G_{y}}{2\left(E G-F^{2}\right)}, \\
& B=\frac{2 G E_{y}+2 F F_{y}-3 F G_{x}-E G_{y}}{2\left(E G-F^{2}\right)}, \\
& C=\frac{G E_{x}+3 F E_{y}-2 F F_{x}-2 E G_{x}}{2\left(E G-F^{2}\right)}, \\
& D=\frac{F E_{x}+E E_{y}-2 E F_{x}}{2\left(E G-F^{2}\right)} .
\end{aligned}
$$

Conversely, if the differential equation of the assigned system of curves is of form (10), and if the functions $A, B, C, D$ are so related that the partial differential equations (11) for $E, F, G$ are consistent, then the problem proposed admits of solution, and the linear elements (8) of the surfaces in question are found by integrating equations (11).

The generalized Beltrami problem admits of solution when, and only when, the differential equation of the assigned system of curves is of form (10) and the partial differential equations (11) are consistent. The determination of the corresponding surfaces depends upon the integration of the set of equations (11).

The original investigation of BeLtram was for the straight lines $y^{\prime \prime}=0$, in which case $A=B=C=D=0$; equations (11) are then consistent, and the surfaces obtainea are those of constant curvature. The case of any linear system (2) may be treated in the same way, but the discussion of (11) becomes then very complicated. * We have already seen however, that the solutions are simply the surfaces of constant curvature. New classes of surfaces present themselves when the system of curves is quadratic, i. e., when the parameters $\lambda, \mu$ in (1) enter in the second degree. This seems to be the next case to investigate in detail.

Columbia University, New York.

${ }^{*}$ Cf. Dr. SteckeR's articles cited above. 